\title{
Different Subsidies' Impact on Equilibrium Decision-making of Closed-loop Supply Chain
}

\author{
Liurui Deng ${ }^{1}$, Wei Li ${ }^{2,3}$, Zilan Liu $^{1}$ \\ ${ }^{1}$ College of Economics and Management, Hunan Normal University (China) \\ ${ }^{2}$ College of Economic and Trade, Hunan University (China) \\ ${ }^{3}$ Hunan Key Laboratory of Logistics Information and Simulation Technology (China) \\ 676352990@qq.com,liwei923@foxmail.com,liuzilan@bunnu.edu.cn
}

Received: April 2014

Accepted: September 2014

\section{Abstract:}

Purpose: An electronic product recycling not only needs the waste treatment company, but also involves recycler and remanufacturer. It is significance for increasing subsidy modes and promoting waste recycling to research the effect of government subsidies for recycler and remanufacturer on the equilibrium of closed-loop supply chain.

Design/methodology/approach: In view of service competition, we build a Stackelberg model which contains an original manufacturer, a recycler and remanufacturer and further obtain the equilibrium decision of closed-loop supply chain. Based on this strategy model, we analyze how two different subsidies to utilization rate of raw materials, the service level and the demand of product affect on the terminal decision.

Findings: We compared the two different subsidy ways in closed-loop supply chain. Subsidy to utilization rate of raw materials has no effect on the whole market demand for electronic product; and subsidy on utilization rate of raw materials has no effect on the whole market demand for electronic product.

Research implications: Under the conditions in which government can observe the information of raw material utility and remanufacturing service level, if government decides to 
expand the market size, it could subsidize remanufacturer for different service level; Under the conditions in which government can observe the information of raw material utility and remanufacturing service level, if government want to improve utilization rate of raw materials, stimulate sustainable development of industries of electronic product, it can subsidizes recycler based on different utilization rate of raw materials.

Originality/value: We analyzed the closed-loop supply chain equilibrium, compared the effects on it under different subsidy mechanism and offered some advises in order to provide scientific support to promote electronic products recycling.

Keywords: subsidy, service, closed-loop supply chain, utilization rate of raw materials, decision-making

\section{Introduction}

In the Provisions on the Collection and Management of Funds for the Disposal of WasteElectrical and Electronic Products issued in 2012, the twentieth clause refers to setting quotas on subsidies to the company according to the quantity ofWaste Electrical and Electronic Products treatment, which indicates that the subsidies is very important for these companies. Because Electronic products recycling not only needs the waste treatment company, but also involves recycler and remanufacturer, so it is significance for increasing subsidy modes and promoting waste recycling to research the effect of government subsidies for recycler and remanufacturer on the equilibrium of closed-loop supply chain.

Two aspects of research have been done on the waste electronic products in the closed loop supply chain; one is about the Electronic products recycling policy, for example, Boks, Nilsson and Masui (1998) have described the collection, dismantling and disposal process of waste electrical and electronic product in West Europe, Japan and US and compared laws and regulations on recycling, landfill and incineration, payment, responsibility, division of recycling among different countries. Nagel, Nilsson and Boks (1999) introduced policies used to construct recycling system of waste electrical and electronic product in Germany, Dutch and Switzerland and summarized recycling cost of various electrical apparatus under different system in different countries. Stevels (2001) researched structure, principles and charging mode of management organization of waste electronic product - NVMP in Dutch. Since Waste Electrical and Electronic Equipment Directive, WEEE Directive has been issued by EU in Feb. 13,2003 , the study on policy of the waste electronic product treatment gradually reduce, then Scott and Supriya constructed the model of a two-echelon closed-loop supply chain based on the EU recycling law of waste electrical and electronic product, and they discussed the impact of competitive strategy and recycling policy on remanufacturing and studied effect of recycling subsidy and government recycling on remanufacturing competitive. The other aspect about 
optimal decision in remanufacturing of waste electronic increased. Ferguson and Toktay analyzed competition between origin manufacturer and remanufacturer, critical condition under which manufacturer decides to remanufacture or not, profit loss and manufacturer entries strategy based on duopoly (Ferguson \& Toktay, 2006). Wang Wenbing etc. studied design of incentive mechanism guided by government in the Electronic products reverse supply chain. They come to the conclusion that government incentive mechanism accelerate recycling rate (Wen-bin \& Qing-li, 2010). Ji Guojun etc. explored decision-making problem of the remanufacturing supply chain under the recycling regulations constrains (Ji \& Huang, 2010) . Xiong Zhongkai investigates coordination mechanism of closed-loop supply chain under patent protection (Xiong, Shen \& Peng, 2011). Wang Wenbing and Da Liqing using recycler profit function studied collection and pricing in closed-loop chain when retailer and the third party are responsible for collection (Wang \& Da, 2010). Li Feng has researched the pricing model and coordination of reverse supply chain in the case of imperfect information and got the optimal pricing contract in which coordination has been achieved ( Li, Sun \& Da, 2009). Zhang Xumei analyzed recycling benefits when manufacturer and recycler coordinate with each other (Zhang \& Huang, 2013). According to Atasu's result, the value of remanufacturing product is perceived lower than new product, two products possess different cost and services competition in terms of remanufacturing and manufacturing process, hence, remanufacturer needs to promote sales through their services (Atasu, Sarvary \& Van Wassenhove, 2008). Cheng-Han Wu studied all members' decision-making problems in the two-echelon closed-loop supple chain with pricing and services competition based on Stackelberg game model, and creatively constructed profit model and so on (Wu, 2012).

With the rapid development of the third industry, services can be traded as an independent product, as well as accessories of the visible product. Service plays a major role throughout all industries (Chen, Liu \& Yuan, 2010; Meng \& Cai, 2009). In the closed-loop supply chain of waste electronic product recycling, the service level of remanufacturing and new product affects the whole market demand and chain decision making. The informed research concentrated on the subsidy for the treatment quantity of the waste electronic product rather than the service level and utilization rate of raw materials hasn't been published. As the development of information and data technology, the information becomes more transparent. Since government can not only observe the price of remanufacturing electronic product, but also the information about supply chain-decision making such as service level and utilization rate of raw materials ( $\mathrm{Wu}, 2012$ ), the subsidy mechanism could be designed on the base of service level and utilization rate of raw materials of remanufacturing product. In this study, based on government subsidy according to decision-making variables of recycler and remanufacturer including utilization rate of raw materials and service level, we have constructed non coordination game model, analyzed the closed-loop supply chain equilibrium, compared the effects on it under different subsidy mechanism and offered some advises in order to provide scientific support to promote electronic products recycling. 


\section{Model}

Original manufacturer produce new product and remanufacturer produce remanufactured product. Although these two products are functionally the same, consumer's willing to pay is different due to their different values. We suppose that recycler collect waste electronic product, remanufacturer only produce remanufactured product and original manufacturer just produce new product. Recycler determines optimal utilization rate of raw materials, original determines servicers' level of new product and remanufacturer decides service level of remanufactured product.

Hypothesis:

Hypothesis 1: All chain members' decisions must be prior to the selling season ( $\mathrm{Wu}$, 2012).

Hypothesis 2: Before the start of the selling season, the price and service information for the products is announced to the market (Wu, 2012).

Hypothesis 3: All chain members are independent, risk-neutral and profit-maximizing (Wu, 2012).

Hypothesis 4: The chain members choose their decisions sequentially in a Stackelberg game.

Hypothesis 5: Both products price $\mathrm{p}$ decided by two manufacturers are constant.

Hypothesis 6: Let $\tau, 0 \leq \tau \leq 1$, be the recycler's decision-making variable, which denote utilization rate of raw materials. Obviously, recycling cost is increasing and remanufacturing cost is decreasing in $\tau$.

Hypothesis 7: there are two government subsidy mechanisms: one is subsidized for utilization rate of raw materials and the other for remanufacturer service level.

In view of these hypothesizes, we define liner demand function of service level. Let subscript $j$ take the values of $r$ and $n$, denoting the remanufactured and new product, respectively. Let $s_{j}$ denote service level of manufacturer $j, p$ denote two products the same price and $\alpha_{j}$ describes the market base parameterizing the potential market size for product $j$ when price at zero and no service is offered. We believe that $\beta_{\mathrm{p}}$ and $\beta_{S}$ represent the prices and service elasticity, respectively. Let $d_{j}$ denote the market demand for product $j, j \in\{r, n\}$ We define the general demand function that captures both product and service competition as follow:

$$
d_{j}=a_{j}-\beta_{p} p+\beta_{s} s_{j}-\gamma_{s}\left(s_{k}-s_{j}\right) ; \alpha_{j}, \beta_{\mathrm{p}}, \beta_{s}>0 ; \gamma_{s} \geq 0, j \in\{\mathrm{r}, \mathrm{n}\}, k=\{\mathrm{r}, \mathrm{n}\} \backslash j,
$$


We denote that $d_{j}$ is upward sloping along its service and $\gamma_{s}$ measures the service competition. Heightening $s_{j}$ by one unit will attract $\gamma_{s}+\beta_{s}$ more customers with demand $d_{j}$ if other parameters are equal.

The whole decision-making process is as follow: Recycler collects used product according to remanufacturer demand, which means that the collection quantity is equal to demand for remanufactured product. Remanufacturer pays $v$ for one unit waste electronic product to recycler. The unit collection cost of waste product is $\frac{b \tau^{2}}{2}$. That is, the collection cost becomes higher as $\tau$ increase. Because recycler is risk-neutral, he has no risk cost. The new product manufacturer carries the production cost and service cost. The total production cost is $\operatorname{cd}_{\mathrm{n}}$, and the service cost is $\left(m s_{n}^{2}\right) / 2$, where $c$ being a unit of production cost and $m$ is the ultimate cost of service. The remanufacturer carries original raw material cost and remanufacturing cost for unit remanufactured product, which are described as $c(1-\tau)$ and $c_{r} \tau$ respectively, where $\tau(0 \leq \tau \leq 1)$ can be considered the portion of recovered materials used to produce a unit product by the manufacturer. $\mathrm{v}$ is the cost of unit recycling product.Assume the cost savings from remanufacturing is a constant and let $\delta \equiv c-c_{r}>0$. The unit remanufacturing cost with returned items can be expressed as $c(1-\tau)+c_{r} \tau=c-\delta \tau(\mathrm{Wu}, 2012)$.

\section{Different Subsidies' Impact on Equilibrium Decision-making of Closed-loop Supply Chain}

\subsection{Equilibrium decision-making under subsidy to utilization rate of raw materials}

If government subsidizes recycler according to utilization rate of raw materials, let $T_{t}$ be Subsidy coefficient, and the subsidy be $\tau T_{t}$. Therefore, the recycling profit function is ( $\mathrm{Wu}$, 2012):

$$
\prod^{R}=v d_{r}-\frac{b \tau^{2}}{2}+\tau T_{t}
$$

The original manufacturer profit function is $(\mathrm{Wu}, 2012)$ :

$$
\prod_{n}^{M}=(p-c) d_{n}-\frac{m s_{n}^{2}}{2}
$$

There manufacturer profit function is (Wu, 2012).:

$$
\prod_{r}^{M}=(p-c+\delta \tau-v) d_{r}-\frac{m s_{r}^{2}}{2}
$$

Now we examine chain members' decision by maximizing their profits in (1)-(3) 
Proposition 1: When government subsidize for utilization rate of raw materials, the closedloop supply chain can reach equilibrium decisions $\tau_{t}{ }^{*}, s_{n t}{ }^{*}$ and $s_{r t}{ }^{*}$.

Focusing on the impact of service levels on the original manufacturer and remanufacturer response functions, we derive the differential of $\prod_{j}^{M}$ with respect to the service level, by solving the first conditions $d \prod_{r}^{M} / d s_{r}=0 ; d \prod_{n}^{M} / d s_{n}=0$, we obtain

$$
s_{r}^{b}=\frac{\Lambda_{s}}{m}(p-c+\delta \tau-v) ; \quad s_{n}^{b}=\frac{\Lambda_{s}}{m}(p-c)
$$

Note that $\Lambda_{s} \equiv \beta_{s}+\gamma_{s}>0$ is the overall effect of the service on its demand.

In (4), we find that $s_{n}{ }^{b}$ is independent of $\tau$; so ${s_{n t}}^{*}=s_{n}{ }^{b}$

With the best response functions for service, and service level in (4), the remanufacturer's best response in terms of his utilization rate of raw materials can be obtained by solving $\tau_{t}^{*} \in \arg \max _{\tau} \prod_{r}^{M}\left(\tau \mid s_{r}^{b}, s_{n}^{b}\right)$, i.e., $d \prod^{R} / d \tau=0$ which yields the equilibrium remanufacturing effort:

$\tau_{t}^{*}=\frac{\left(T_{t}+\frac{v \delta \Lambda_{s}^{2}}{m}\right)}{b}$

With $\tau_{t}^{*}=\frac{\left(T_{t}+\frac{v \delta \Lambda_{s}^{2}}{m}\right)}{b}$, we can get equilibrium service levels ${s_{n t}}^{*}$ and $s_{r t}{ }^{*}$ with $\gamma_{s} \neq 0$ which presents the presence of service competition.

$$
s_{r t}^{*}=\frac{\Lambda_{s}}{m}\left[p-c+\frac{\delta T_{t}+\frac{v \delta^{2} \Lambda_{s}^{2}}{m}}{b}-v\right] ; s_{n t}^{*}=\frac{\Lambda_{s}}{m}(p-c)
$$

With the first differential of equilibrium decision respect to $T_{t}$, we can obtain:

$$
d \tau_{t}^{*} / d T_{t}=\frac{1}{b}>0 ; \quad d s_{r t}^{*} / d T_{t}=\frac{\Lambda_{s} \delta}{b m} ; d s_{n t}^{*} / d T_{t}=0
$$

Proposition 1 is proved.

Conclusion 1: when subsidy coefficient increases one unit, recycling decision-making $\tau_{t}{ }^{*}$, increases $\frac{1}{h}$ unit, remanufacturer equilibrium ${s_{r t}}^{*}$ increases $\frac{\Lambda_{s} \delta}{b m}$ unit and the original manufacturer $s_{n t}{ }^{*}$ do not change. 
From conclusion 1 we can see that subsidy for utilization rate of raw materials has positive effect on the remanufacturing service level, and has no effect on manufacturing service level.

Proposition 2: when subsidies to utilization rate of raw materials, we can obtain optimal new product demand $d_{n t}{ }^{*}$ and remanufactured product demand $d_{r t}{ }^{*}$

Applying equilibrium decision-making of all members in close-cycle supply to demand function, it is easy to get that

$$
\begin{gathered}
d_{n t}^{*}=\frac{b m^{2}\left(a_{n}-p \beta_{p}\right)+m\left[-b(c-p) \beta_{s}+\left(b v-T_{t} \delta\right) \gamma_{s}\right] \Lambda_{s}-v \delta^{2} \gamma_{s} \Lambda_{s}^{3}}{b m^{2}} \\
d_{r t}^{*}=\frac{b m^{2}\left(a_{r}-p \beta_{p}\right)+m\left[\left(-b(c-p+v)+T_{t} \delta\right) \beta_{s}+\left(-b v+T_{t} \delta\right) \gamma_{s}\right] \Lambda_{s}+v \delta^{2} \Lambda_{s}^{4}}{b m^{2}}
\end{gathered}
$$

Then we derive the first differential of demand with respect to $T_{t}$, we can obtain:

$$
d d_{n t}^{*} / d T_{t}=-\frac{\delta \gamma_{s} \Lambda_{s}}{b m}<0 ; \quad d d_{r t}^{*} / d T_{t}=\frac{\delta \gamma_{s} \Lambda_{s}}{b m}>0
$$

Conclusion 2: when $T_{t}$ increases one unit, new product demand $d_{n t}{ }^{*}$ will decrease $\frac{\delta \gamma_{s} \Lambda_{s}}{h m}$ unit, remanufactured product $d_{r t}{ }^{*}$ demand will increases $\frac{\delta \gamma_{s} \Lambda_{s}}{b m}$ unit.

With conclusion 2, we can show that the decreasing demand for new product is equal to increasing demand for remanufactured product whether the $T_{t}$ increases or decreases. That is, the subsidy to utilization rate of raw materials has no effect on the whole market demand for electronic product.

\subsection{Subsidy to remanufacturing service level}

When government subsidize in terms of remanufacturing service level, the recycling profit function $(\mathrm{Wu}, 2012)$ is:

$$
\prod^{R}=v d_{r}-\frac{b \tau^{2}}{2}
$$

The original manufacturer profit function is $(\mathrm{Wu}, 2012)$ :

$$
\prod_{n}^{M}=(p-c) d_{n}-\frac{m s_{n}^{2}}{2}
$$


If government subsidizes recycler according to remanufacturing service level, set $T_{r}$ be Subsidy coefficient, and the subsidy is $s_{r} T_{t}$. Therefore remanufacturer profit function $(\mathrm{Wu}, 2012)$ is:

$$
\prod_{r}^{M}=(p-c+\delta \tau-v) d_{r}-\frac{m s_{r}^{2}}{2}+T_{r} s_{r}
$$

Now we examine chain members' decision by maximizing their profits in (11)-(13)

Proposition 3: When government subsidize based on remanufacturing service level, the closed-loop supply chain can reach equilibrium decisions, $\tau_{r}{ }^{*}, s_{r r}{ }^{*}$ and $s_{n r}{ }^{*}$.

Focusing on the impact of service levels on the original manufacturer and remanufacturer response functions, we derive the differential of $\prod_{j}^{M}$ with respect to the service level, by solving the first conditions $d \prod_{r}^{M} / d s_{r}=0 ; d \prod_{n}^{M} / d s_{n}=0$, we obtain

$$
s_{r}^{b}=\frac{\Lambda_{s}}{m}(p-c+\delta \tau-v)+\frac{T_{r}}{m}, \quad s_{n}^{b}=\frac{\Lambda_{s}}{m}(p-c)
$$

Note that $\Lambda_{s} \equiv \beta_{s}+\gamma_{s}>0$ is the overall effect of the service on its demand.

In (14), we find that $s_{n}{ }^{b}$ is independent of $\tau$; so ${s_{n}}^{*}=s_{n}{ }^{b}$

With the best response functions for service, and service level in (14), the remanufacturer's best response in terms of his utilization rate of raw materials can be obtained by solving $\tau_{t}^{*} \in \arg \max _{\tau} \prod_{r}^{M}\left(\tau \mid s_{r}^{b}, s_{n}^{b}\right)$, i.e., $d \prod^{R} / d \tau=0$ which yields the equilibrium remanufacturing effort: $\tau_{r}^{*}=\frac{v \delta \Lambda_{s}^{2}}{b m}(15)$

With $\tau_{r}^{*}=\frac{v \delta \Lambda_{s}^{2}}{h m}$, we can derive equilibrium service levels $s_{r}{ }^{*}, s_{n}{ }^{*}$ with $\gamma_{s} \neq 0$ which presents the presence of service competition:

$$
s_{r r}^{*}=\frac{\Lambda_{s}}{m}\left(p-c+\frac{v \delta^{2} \Lambda_{s}^{2}}{h m}-v\right)+\frac{T_{r}}{m}, \quad s_{n r}^{*}=\frac{\Lambda_{s}}{m}(p-c)
$$

Proposition 3 is proved.

Then we derive the first differential of equilibrium decision with respect to $T_{r}$, we can obtain:

$$
d s_{r r}^{*} / d T_{r}=\frac{1}{m} ; d \tau_{r}^{*} / d T_{r}=0 ; d s_{n r}^{*} / d T_{r}=0
$$


Conclusion 3: when subsidy coefficient increases one unit, remanufacturer equilibrium $S_{r r}{ }^{*}$ increases $\frac{1}{m}$ unit, but recycling decision-making $\tau_{r}{ }^{*}$ and the original manufacturer ${S_{n r}}^{*}$ have not been influenced.

From conclusion 3, it is easy to see that remanufacturing service level has positive effect on the remanufacturing service level, but has no effect on utilization rate of raw materials and manufacturing service level.

Proposition 4: when subsidize according to remanufacturing service level, we can obtain optimal new product demand $d_{n r}{ }^{*}$ and remanufactured product demand $d_{r r}{ }^{*}$

Using the same method as Proposition 2, we can see that

$$
\begin{aligned}
& d_{n r}^{*}=a_{n}+\frac{-b m\left(m p \beta_{p}+T_{r} \gamma_{s}\right)+b m\left[(-c+p) \beta_{s}+v \gamma_{s}\right] \Lambda_{s}-v \delta^{2} \gamma_{s} \Lambda_{s}^{3}}{b m^{2}} \\
& d_{r r}^{*}=\frac{b m^{2}\left(a_{r}-p \beta_{p}\right)+b m T_{r} \Lambda_{s}-b m\left[(c-p+v) \beta_{s}+v \gamma_{s}\right]+v \delta^{2} \Lambda_{s}^{4}}{b m^{2}}
\end{aligned}
$$

Proposition 4 is proved.

Then we derive the first differential of demand with respect to $T_{r}$, we can achieve the result that

$$
d d_{n r}^{*} / d T_{r}=-\frac{\gamma_{s}}{m} ; d d_{r r}^{*} / d T_{r}=\frac{\Lambda_{s}}{m}
$$

Conclusion 4: when $T_{r}$ increases one unit, new product demand $d_{n r}{ }^{*}$ will decrease $\frac{\gamma_{s}}{m}$ unit, remanufactured product $d_{r r}{ }^{*}$ demand will increases $\frac{\Lambda_{s}}{m}$ unit.

From conclusion 4, we can show that the decreasing demand for new product is equal to increasing demand for remanufactured product. Whether the $T_{t}$ increases or decreases, we can show that the subsidy on utilization rate of raw materials has no effect on the whole market demand for electronic product. 


\section{Numerical simulation}

In order to research the influence of different subsidy mechanism on chain members equilibrium decision-making directly, we turn to numerical analyses of above results. We define subsidy to utilization rate of raw materials as mechanism A, and remanufacturing service level as mechanism $B$ for convenience.

A selected set of parameters is as follows: $a_{r}=a_{n}=144, b=60, m=90, p=3, c=1.5, v=$ $0.1, \delta=1, \beta_{\mathrm{p}}=20, \gamma_{s}=15, \beta_{s}=15$

(1) based on mechanism A, we obtain: $\tau_{t}^{*}=\frac{T_{t}^{\prime}+1}{60} ; s_{r t}^{*}=\frac{17}{36}+\frac{T_{t}}{180} ; s_{n t}^{*}=\frac{1}{2}$; $d_{n t}^{*}=\frac{1103}{12}-\frac{T_{t}}{12} ; \quad d_{r t}^{*}=\frac{272}{3}+\frac{T_{t}}{6}$

(2) based on mechanism B, we can obtain: $\tau_{r}^{*}=\frac{1}{60} ; s_{r r}^{*}=\frac{17}{36}+\frac{T_{r}}{90} ; s_{n r}^{*}=\frac{1}{2}$;

$d_{n r}^{*}=\frac{1103}{12}-\frac{T_{r}}{6} ; \quad d_{r r}^{*}=\frac{5063}{60}+\frac{T_{r}}{3}$

When the equilibrium decision-making of $A$ is subtracted by $B$, we can compare the utilization rate of raw materials, service level and demand with figures of two different subsidy mechanisms.

As $\Delta \tau^{*}=\tau_{t}^{*}-\tau_{r}^{*}=\frac{T_{t}}{60}$, materials' utilization rate of $\mathrm{A}, \tau_{r}{ }^{*}$ is larger than $\mathrm{B}$. And, the distinction between them increases $T_{t}$. But it is not influenced by $T_{r}$.

From Figure 1, we can see that when $2 T_{r}=T_{t}, \Delta s_{r}{ }^{*}=0$, which indicates that two service level are equal under different mechanism. If $2 T_{r}>T_{t}, \Delta s_{r}{ }^{*}<0$, the remanufacturing service level of $A$ is higher than $B$ and vice versa.

With Figure 2, it is shown that if $2 T_{r}=T_{t}, \Delta d_{n}{ }^{*}=0$, which indicates that when subsidy coefficient of $\mathrm{A}$ is twice as much as $\mathrm{B}$, two product demands are equal. If $2 T_{r}>T_{t}, \Delta d_{n}{ }^{*}>0$, and vice versa. 


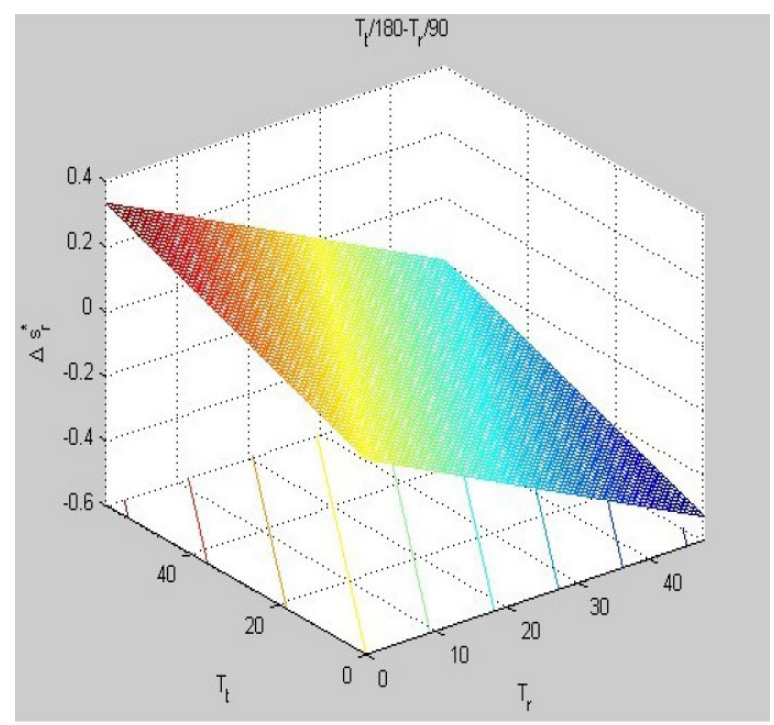

Figure 1. Influences of $T_{t}$ and $T_{r}$ on $\Delta s_{r}{ }^{*}$

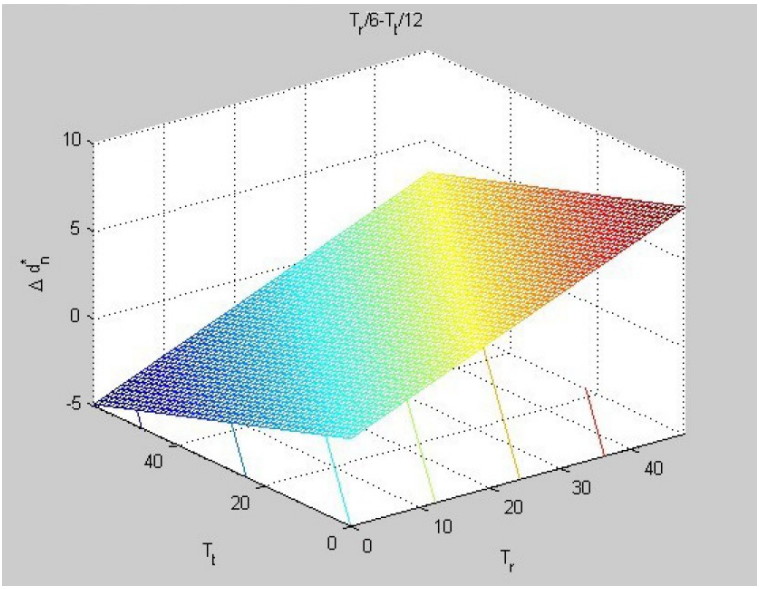

Figure 2. Influences of $T_{t}$ and $T_{r}$ on $\Delta d_{n}{ }^{*}$

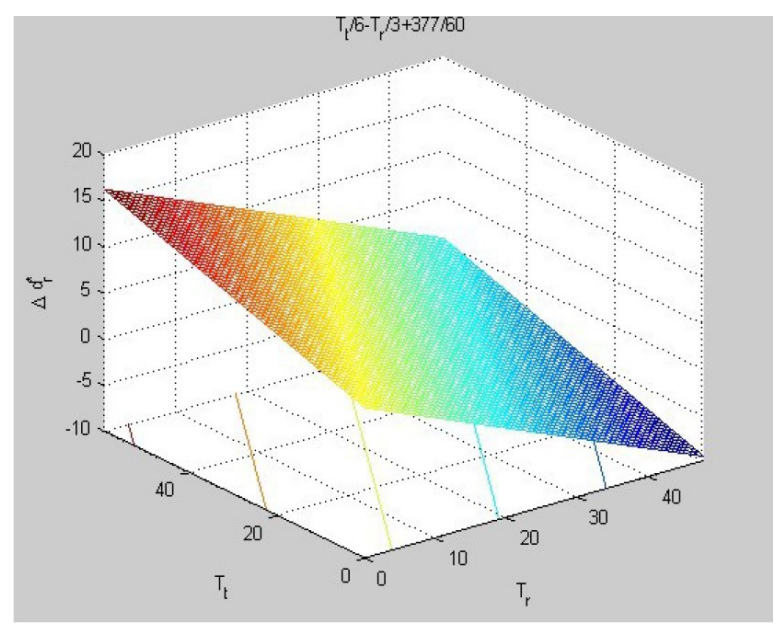

Figure 3. Influences of $T_{t}$ and $T_{r}$ on $\Delta d_{r}{ }^{*}$ 
Noticing $\Delta d_{r}^{*}=\frac{377}{60}+\frac{T_{t}}{6}-\frac{T_{r}}{3}$, we can get the result that if $20 T_{r}-10 T_{t}=377, \Delta d_{r}{ }^{*}=0$. If $20 T_{r}-10 T_{t}>377, \Delta d_{r}{ }^{*}<0$. If $20 T_{r}-10 T_{t}<377, \Delta d_{r}{ }^{*}>0$. In Figure 3, We can observe that with the distinction between twice subsidy coefficient of $A$ and subsidy coefficient of $B$ increasing, first, demand for remanufactured product of $A$ is lager than $B$, then two demand are the same and finally $B$ is larger than $A$.

\section{Solutions and Advises}

\subsection{Expand the size of the market}

When government subsidizes recycler, decreasing demand for new product is the same with the increasing demand for remanufactured product, so the total demand do not change and the size of market keep the same. In the view of economic benefits, demand for product, consumption level of consumer and social utility level keep constant. Government will not implement subsidy policy as a rational individual. When government subsidizes remanufacturer, the decreasing demand for new product is smaller than increasing demand for remanufactured product. So the total demand increases, the market size expand, consumption level increases and the whole social utility increases which shows that government interferes play a positive role. Therefore, under the conditions in which government can observe the information of raw material utility and remanufacturing service level, if government decides to expand the market size, it could subsidize remanufacturer for different service level.

\subsection{Improve utilization rate of raw materials}

When government subsidizes recycler, utilization rate of raw materials is increasing in subsidy coefficient. In the view of sustainable development, the bigger the subsidy coefficient, the higher the utilization rate of raw materials. When government subsidizes remanufacturer, there is no influence of subsidy coefficient on utilization rate of raw materials which shows that utilization rate of raw materials keep constant. Therefore, under the conditions in which government can observe the information of raw material utility and remanufacturing service level, if government want to improve utilization rate of raw materials, stimulate sustainable development of industries of electronic product, it can subsidizes recycler based on different utilization rate of raw materials. 


\section{Conclusion}

There are lots of studies on the effect of subsidy on the electronic products, but the most of them only analyze the case of the constant subsidy rate of one remanufacturing product. However, we research on decision-making on the closed-loop supply chain of electronic products under government's subsidy for the raw material and remanufacturing service level. In order to compare the influence on different subsidy modes of closed-loop supply chain, we build a Stackelberg model which contains an original manufacturer, a recycler and remanufacturer. We solve the game model by optimization method. Considering the equilibrium solutions are too complicated, we analyze the effects on different subsidy for equilibrium utilization rate of raw materials, service level of new product and remanufactured product and demand for them by numerical simulation. The results show that: (1) both two subsidy modes can increase remanufactured product quantity and remanufacturing service level; (2) both two subsidy modes have no effect on the manufacturing service level; (3) the total demand could not be affected by subsidy to utilization rate of raw materials, but can be influenced by subsidy to remanufacturing service level.

Our work involves three parties: original manufacturer, remanufacturer and recycler. In the future, we can study the complete closed-loop supply chain including original manufacturer, remanufacturer, retailer and recycler and analyze the effect of price, service competition with various subsidies on decision-making in closed-loop supply chain. The product design also can be considered when we analyzed influence of the product's dismantled ability on decisionmaking.

\section{Acknowledgments}

This study was supported by National Natural Science Foundation of China (71201051), Doctoral Research Fund of Hunan Normal University (Grant No. 2014BQ11), Young Talents Training Plan of Hunan Normal University (2014YX04), general project of the ministry of education on humanities and social science research (11YJC790084), Research fund for the Doctoral Program of higher education (20110161120032) and philosophy social science fund of Hunan province (2010YBA048). 


\section{References}

Atasu, A., Sarvary, M., \& Van Wassenhove, L.N. (2008). Remanufacturing as a Market Strategy. Management Science, 54, 1731-1746. http://dx.doi.org/10.1287/mnsc.1080.0893

Boks, C., Nilson, J., \& Masui, K. (1998). An international comparison of product end-of-life scenarios and legislation for consumer electronics. IEEE International Symposium on Electronics and the Environment, 19-24.

Chen, Z.P., Liu, Y.B., \& Yuan, P. (2010). Service Innovation: Features and the Dispensability of Police. China Soft Science, 12, 43-49.

Ferguson, M.E., \& Toktay, L.B. (2006). The Effect of Competition on Recovery Strategies. Production And Operations Management, 15, 351-368.

http://dx.doi.org/10.1111/j.1937-5956.2006.tb00250.x

Ji, G.J., \& HUANG W.W. (2010). Remanufacturing Supply Chain Decision under the Constraints of Take-back Laws. Systems Engineering-theory \& Practice, 30, 1355-1362

Li, F., Sun, H. \& Da, Q.L. (2009). Study on the Pricing and Coordinating Mechanism with Incomplete Information in Remanufacturing Reverse Supply Chain. Chinese Journal of Management Science, 17, 72-80.

Meng, Y.H., \& Cai, H.B. (2009). Economic Laissez-Faire and Regulation Policy in Liberalization of Trade in Service. Nankai Economic Studies, 6, 100-111.

Nagel, C., Nilsson, J., \& Boks, C. (1999). European End-of-Life Systems for Electrical and Electronic Equipment. Proceedings. EcoDesign: First International Symposium on Environmentally Conscious Design and Inverse Manufacturing. Tokyo, 9, 197-202.

Stevels, A. (2001). Experiences with the Take-back of White and Brown Goods in the Netherlands [C]. IEEE Proceedings of 2nd International Symposium Environmentally Conscious Design and Inverse Manufacturing Tokyo, 489-493.

http://dx.doi.org/10.1109/ECODIM.2001.992405

Wang, W.B., \& Da, Q.L. (2010). The Study on Collection and Pricing for Closed-loop Supply Chain with Retailer and the Third Party Collecting. Journal of Industrial Engineering and Engineering Management, 2, 130-134.

Wen-bin, W., \& Qing-li, D.A. (2010). Study on Premium and Penalty Mechanisms for the Electronic Product Reverse Supply Chain Considering the Leading of Government. Chinese Journal of Management Science, 18, 62-67. 
Wu, C.H. (2012). Price and Service Competition between New and Remanufactured Products in a Two-echelon Supply Chain. International Journal of Production Economics, 140, 496-507. http://dx.doi.org/10.1016/j.ijpe.2012.06.034

Xiong, Z.K., Shen, C.R., \& Peng, Z.Q. (2011). Closed-loop Supply Chain Coordination Research with Remanufacturing under Patent Protection. Journal of Management Sciences in China, 14, 76-85.

Zhang, X.M., \& Huang, C.X. (2013). Decision-making Mechanism for Knowledge Sharingin Reverse Supply Chain Chinese. Journal of Management, 10, 233-237.

Journal of Industrial Engineering and Management, 2014 (www.jiem.org)

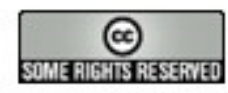

Article's contents are provided on a Attribution-Non Commercial 3.0 Creative commons license. Readers are allowed to copy, distribute and communicate article's contents, provided the author's and Journal of Industrial Engineering and Management's names are included. It must not be used for commercial purposes. To see the complete license contents, please visit http://creativecommons.org/licenses/by-nc/3.0/. 